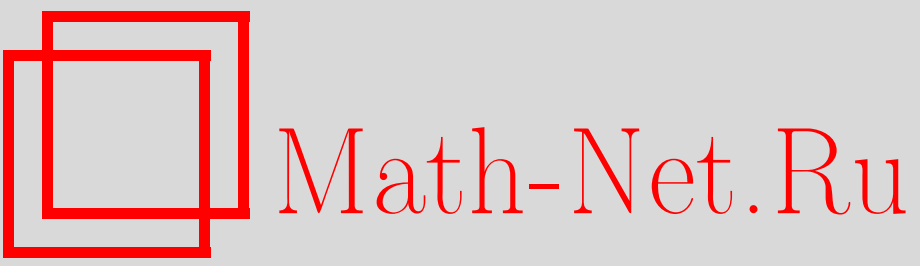

В. К. Петров, Простая модель для исследования поведения кирального конденсата при конечных температурах на предельно анизотропной решетке, ТM $\Phi$, 1999, том 121, номер 3, 436-449

DOI: https://doi.org/10.4213/tmf821

Использование Общероссийского математического портала Math-Net.Ru подразумевает, что вы прочитали и согласны с пользовательским соглашением

http://www.mathnet.ru/rus/agreement

Параметры загрузки:

IP : 54.81 .137 .203

26 апреля 2023 г., 13:00:52 
ТЕОРЕТИЧЕСКАЯ

И МАТЕМАТИЧЕСКАЯ

ФИЗИКА

Том 121, № 3

декабрь, 1999

(C) 1999 г.

\author{
В.К. Петров*
}

\title{
ПРОСТАЯ МОДЕЛЬ ДЛЯ ИССЛЕДОВАНИЯ \\ ПОВЕДЕНИЯ КИРАЛЬНОГО КОНДЕНСАТА ПРИ КОНЕЧНЫХ ТЕМПЕРАТУРАХ НА ПРЕДЕЛЬНО АНИЗОТРОПНОЙ РЕШЕТКЕ
}

В рамках простой решеточной модели квантовой хромодинамики вычислен фермионный детерминант. Поведение кирального конденсата исследуется в области асимптотически больших $(m \gg T)$ и асимптотически малых $(m \ll T)$ масс кварков.

\section{1. ВВЕДЕНИЕ}

Вклад фермионов в решеточных калибровочных теориях на протяжении многих лет исследовался как аналитически, так и численно. Важнейшим аспектом таких исследований была возможность сохранения киральной симметрии при переходе к континуальному пределу. В последние годы удалось преодолеть ряд серьезных технических проблем, связанных с рассмотрением динамических фермионов. Однако численные вычисления в теории с динамическими фермионами и малым решеточным шагом все еше остаются дорогостоящими. Поэтому даже грубые модели, допускающие аналитическое решение, могут оказаться весьма полезными.

Хотя технические трудности не позволяют решить задачу точно, сушествует ряд приближений, которые, как нам представляется, в достаточной степени отражают сущность рассматриваемых процессов. В этой работе вклад динамических фермионов исследуется в рамках простой модели [1], основным предположением которой является исключение из действия всех членов, пропорциональных $1 / \xi^{1)}$. Такого рода приближения в той или иной форме использовались в решеточных моделях достаточно давно. Так, в работах $[2,3]$ рассматривалось приближение, в котором не учитывается магнитная часть действия. В этом смысле выбранное приближение сходно с приближением сильной связи. Более того, эффективное действие совпадает с полученным в работах $[4,5]$ в

\footnotetext{
1) Параметр анизотропии $\xi$ определяется как $\xi \simeq a_{\sigma} / a_{\tau}$, где $a_{\tau}$ и $a_{\sigma}$ - решеточные шаги соответственно во временном и пространственном направлениях.
}

${ }^{*}$ Институт теоретической физики им. Н.Н. Боголюбова Национальной академии наук Украины, Киев, Украина. E-mail: petrov@777.com.ua 
области, где константа связи $g$ велика $\left(g^{2} \gg 1\right)$. Однако на анизотропной решетке $\xi \gg 1$ с $N_{\tau} \gg 1$ (где $N_{\tau}$ - протяженность решетки во временном направлении) условие $g^{2} \gg 1$ излишне и область слабой связи не является недоступной, что позволяет изучить континуальный предел в рамках данной модели.

В предыдущей работе [1] был изучен чисто глюонный сектор квантовой хромодинамики (КХД) в рассматриваемом приближении. На экстремально больших решетках $\left(N_{\tau} \rightarrow \infty\right)$ модель допускает аналитическое решение и позволяет найти выражение для бегушей константы связи в области $a_{\tau} \rightarrow 0$. Оценки, полученные для бета-функции Каллана-Симанзика, не являются неожиданными, но едва ли близки к реальности, т.к. приводят к тривиальной асимптотической свободе $g^{2} \sim a_{\tau}$. Чтобы приблизить модель к реальной картине взаимодействующих кварков и глюонов, естественно включить в рассмотрение динамические фермионы. И хотя это вряд ли сделает модель вполне реалистичной, некоторые результаты, к которым приводит включение динамических фермионов в рассматриваемой модели, могут оказаться справедливыми и в точной теории.

Мы будем работать в решеточной формулировке КХД с вильсоновскими фермионами. Этот выбор продиктован исключительно соображениями удобства. Как известно, каждая из формулировок (вильсоновская и когут-сасскиндовская) имеет свои преимушества (подробный анализ дан в работах $[6,7]$ ). Предполагается, что результаты, полученные в разных формулировках, должны совпадать в континуальном пределе. В соответствии с теоремой Нильсена-Ниномии [8] невозможно снять вырождение фермионного спектра без нарушения киральной инвариантности. В случае вильсоновских фермионов киральная симметрия нарушена явно вильсоновским членом, введенным для подавления вклада зеркальных фермионов. Это затрудняет изучение спонтанного нарушения киральной симметрии в теории с вильсоновскими фермионами. В когут-сасскиндовской формулировке массу фермионов можно устремить к нулю, но в этом подходе возникают трудности с определением киральных преобразований в континуальном пределе [9].

\section{2. ФЕРМИОННЫЙ ДЕТЕРМИНАНТ В ПРОСТОЙ МОДЕЛИ}

Для фермионной части действия используется выражение, предложенное в [10] (см. также [11]),

$$
-\mathcal{S}_{\mathrm{F}}=n_{f}\left(a^{3} \sum_{x, x^{\prime}} \bar{\psi}_{x^{\prime}} D_{x^{\prime} x}^{0} \psi_{x}+\frac{a^{3}}{\xi} \sum_{n=1}^{d} \sum_{x, x^{\prime}} \bar{\psi}_{x^{\prime}} D_{x^{\prime} x}^{n} \psi_{x}\right),
$$

где $a \equiv a_{\sigma}, n_{f}$ - число ароматов,

$$
\begin{aligned}
& D_{x^{\prime} x}^{n}=\frac{r-\gamma_{n}}{2} U_{n}(x) \delta_{x, x^{\prime}-n}+\frac{r+\gamma_{n}}{2} U_{n}^{\dagger}\left(x^{\prime}\right) \delta_{x, x^{\prime}+n}-r \delta_{x^{\prime}, x}, \\
& D_{x^{\prime} x}^{0}=\frac{r-\gamma_{0}}{2} U_{0}(x) \delta_{x, x^{\prime}-0}+\frac{r+\gamma_{0}}{2} U_{0}^{\dagger}\left(x^{\prime}\right) \delta_{x, x^{\prime}+0}-\left(m a_{\tau}+r\right) \delta_{x^{\prime}, x},
\end{aligned}
$$

здесь $\gamma_{\nu}$ - матрицы Дирака $(\nu=0, n ; n=1,2,3,4), \psi_{x}$ - фермионные поля, заданные в узлах решетки $x=(t, \vec{x}), U_{\nu}(x)$ - калибровочные поля, заданные на ребрах $x, x+\nu$, $\dagger$ - эрмитово сопряжение. 
Вильсоновский член с конечным параметром $r(0<r \leqslant 1)$ не меняет существенно вклад обычных фермионов в инфракрасном пределе, однако подавляет вклад зеркальных фермионов, увеличивая их эффективную массу.

Поскольку в выбранном приближении мы пренебрегаем всеми членами порядка $1 / \xi$, фермионное действие в простой модели сводится к $a^{3} \sum_{x} \bar{\psi}_{x, x^{\prime}} D_{x x^{\prime}}^{0} \psi_{x^{\prime}}$. Пространственная часть пропагатора фермионов включает фактор $1 / \xi$ и должна быть отброшена, поэтому в такой модели фермионы едва ли можно считать динамическими в полном смысле этого слова. Тем не менее они приводят к нетривиальному члену в эффективном действии, который мы попытаемся здесь вычислить.

Фермионные поля $\psi_{x}$ могут быть представлены в виде линейной комбинации операторов рождения $\mathbf{a}_{p, \sigma}^{\dagger}$ и уничтожения $\mathbf{a}_{p, \sigma}$ частиц (и соответственно античастиц $\mathbf{b}_{-p,-\sigma}^{\dagger}$, $\left.\mathbf{b}_{-p,-\sigma}\right)$ с 4-импульсами $p$ и проекциями спинов $s$ :

$$
\begin{aligned}
\psi_{x} & =\sum_{p, \sigma}\left(u_{p, \sigma}(x) \mathbf{a}_{p, \sigma}+u_{-p,-\sigma}(x) \mathbf{b}_{-p,-\sigma}^{\dagger}\right) \\
\bar{\psi}_{x} & =\sum_{p, \sigma}\left(\bar{u}_{p, \sigma}(x) \mathbf{a}_{p, \sigma}^{\dagger}+\bar{u}_{-p,-\sigma}(x) \mathbf{b}_{-p,-\sigma}\right) .
\end{aligned}
$$

В стандартной процедуре квантования [12] такие операторы являются переменными интегрирования. Однако их комбинация может быть тоже использована с этой целью при условии, что соответствуюший якобиан перехода не равен нулю. Напомним, что в стандартном представлении

$$
\gamma_{0}=\left(\begin{array}{cc}
1 & 0 \\
0 & -1
\end{array}\right), \quad \gamma_{n}=\left(\begin{array}{cc}
0 & \sigma_{n} \\
\sigma_{n} & 0
\end{array}\right)
$$

проекторы $\left(1 \pm \gamma_{0}\right) / 2$ делят биспиноры $\psi$ на две компоненты $\psi^{( \pm)}$, каждая из которых представляет собой двухкомпонентный спинор

$$
\psi^{(+)}=\frac{1+\gamma_{0}}{2} \psi, \quad \psi^{(-)}=\frac{1-\gamma_{0}}{2} \psi
$$

и является собственным вектором оператора четности

$$
P \psi^{( \pm)}= \pm i \psi^{( \pm)}
$$

Принимая во внимание, что $\left(\delta_{x-\nu, x^{\prime}}\right)^{\dagger}=\delta_{x^{\prime}, x-\nu}=\delta_{x+\nu, x^{\prime}}$ и

$$
\frac{r \pm \gamma_{0}}{2}=\frac{1+\gamma_{0}}{2} \frac{r \pm 1}{2}+\frac{r \mp 1}{2} \frac{1-\gamma_{0}}{2}
$$

можно переписать действие $(1)$ в терминах $\psi^{( \pm)}$:

$$
-\mathcal{S}_{F}=\sqrt{1-r^{2}} n_{f} \sum_{x, x^{\prime}}\left(\bar{\psi}_{x^{\prime}}^{(+)} \Delta_{x^{\prime} x}^{\dagger} \psi_{x}^{(+)}+\bar{\psi}_{x^{\prime}}^{(-)} \Delta_{x^{\prime} x} \psi_{x}^{(-)}\right)
$$


где

$$
\Delta_{x x^{\prime}}=\delta_{\vec{x}, \vec{x}^{\prime}}\left(\frac{e^{a_{\tau} m_{r}} U_{0}(\vec{x}, t)}{2} \delta_{t-1, t^{\prime}}-\frac{e^{-a_{\tau} m_{r}} U_{0}^{\dagger}\left(\vec{x}, t^{\prime}\right)}{2} \delta_{t+1, t^{\prime}}-a_{\tau}\left(m+m_{r}\right) \delta_{t, t^{\prime}}\right),
$$

$$
m_{r} \equiv \frac{1}{a_{\tau}} \operatorname{arth} r
$$

При $a_{\tau} \rightarrow 0$ и $m_{r} \ll 1 / a_{\tau}$ имеем

$$
\frac{m a_{\tau}+r}{\sqrt{1-r^{2}}}=\left(m+m_{r}\right) a_{\tau}+O\left(a_{\tau}^{3}\right) .
$$

Все матрицы $U_{0}(\vec{x}, t)_{\alpha \nu}$ могут быть диагонализованы одновременно, т.е. $U_{0}(\vec{x}, t)_{\alpha \nu}=$ $\delta_{\alpha \nu} U_{0}(\vec{x}, t)_{\alpha \alpha}$. Следовательно, оператор $\Delta_{x x^{\prime}}=\left(\Delta_{x x}\right)_{\alpha}$ в (10) представляет собой набор $N$ матриц $N_{\tau} \times N_{\tau}$ и может быть записан в виде

$$
\Delta=\left(\begin{array}{cccc}
\left(m+m_{r}\right) a_{\tau} & \lambda U_{0}^{\dagger}(\vec{x}, 0) & \cdots & -\lambda(-1)^{B} U_{0}\left(\vec{x}, N_{\tau}-1\right) \\
-\lambda U_{0}(\vec{x}, 0) & \left(m+m_{r}\right) a_{\tau} & \cdots & 0 \\
0 & -\lambda U_{0}(\vec{x}, 1) & \cdots & 0 \\
\cdots & \cdots & \cdots & \cdots \\
0 & 0 & \cdots & \lambda U_{0}^{\dagger}\left(\vec{x}, N_{\tau}-2\right) \\
\lambda(-1)^{B} U_{0}^{\dagger}\left(\vec{x}, N_{\tau}-1\right) & 0 & \cdots & \left(m+m_{r}\right) a_{\tau}
\end{array}\right),
$$

где $\lambda \equiv e^{-m_{r} a_{\tau}} / 2$, а $B=0$ для периодических граничных условий и $B=1$ для антипериодических ${ }^{2)}$. В рассматриваемом простом случае $\operatorname{det} \Delta$ может быть легко вычислен (см. приложение). Таким образом, после интегрирования по $\psi_{x}^{( \pm)}$-полям получаем, что

$$
-\mathcal{S}_{\mathrm{F}}^{\mathrm{eff}}=n_{f} \ln \operatorname{det} \Delta^{\dagger} \Delta=2^{2-2 N_{\tau}} n_{f} \sum_{\alpha} \ln \Xi_{\alpha}^{*} \Xi_{\alpha},
$$

где

$$
\Xi_{\alpha}=M_{N_{\tau}}+(-1)^{B} \frac{e^{\frac{m_{r}}{T}} \Omega_{\alpha}+(-1)^{N_{\tau}} e^{-\frac{m_{r}}{T}} \Omega_{\alpha}^{*}}{2},
$$

здесь $T=\left(N_{\tau} a_{\tau}\right)^{-1}-$ температура,

$$
\Omega_{\alpha} \equiv \exp \left(i \varphi_{\alpha}\right)=\prod_{t=1}^{N_{\tau}-1} U_{0}(\vec{x}, t)_{\alpha \alpha}
$$

- собственные значения поляковских матриц, причем фазы $\varphi_{\alpha}$ удовлетворяют условию

$$
\sum_{\alpha=1}^{N} \varphi_{\alpha}=0
$$

\footnotetext{
2) Условие положительности Остервальда-Шрадера [13] требует наложения антипериодических граничных условий на фермионные поля в континуальной теории. Обобщение условия положительности для вильсоновской формулировки решеточной калибровочной теории дано Остервальдом и Зайлером [14].
} 
а $M_{N_{\tau}}$ дается соотношением

$$
M_{N_{\tau}}= \begin{cases}\operatorname{ch}\left(N_{\tau} \operatorname{arsh} \widehat{m}\right)=T_{N_{\tau}}\left(\sqrt{\widehat{m}^{2}+1}\right), & N_{\tau}=2 k, \\ \operatorname{sh}\left(N_{\tau} \operatorname{arsh} \widehat{m}\right)=i^{-N_{\tau}} T_{N_{\tau}}(i \widehat{m}), & N_{\tau}=2 k+1,\end{cases}
$$

$\widehat{m}=\left(m+m_{r}\right) a_{\tau}$ и $T_{n}(x) \equiv \cos (n \arccos x)$ - полиномы Чебышева порядка $n$ (см. [15, гл. $10, \S 11$, формула (2)]).

Легко показать, что при $a_{\tau} \rightarrow 0$ выражение (16) приводит к соотношению

$$
M_{N_{\tau}} \simeq \begin{cases}\operatorname{ch}\left(\frac{m+m_{r}}{T}\right)+O\left(a_{\tau}^{2}\right), & N_{\tau}=2 k \\ \operatorname{sh}\left(\frac{m+m_{r}}{T}\right)+O\left(a_{\tau}^{2}\right), & N_{\tau}=2 k+1\end{cases}
$$

Следовательно, можно записать в случае антипериодических граничных условий ${ }^{3)}$, что

$$
-\mathcal{S}_{\mathrm{F}}^{\mathrm{eff}}=n_{f} \sum_{\alpha=1}^{N} \ln \left(R^{2}+(-1)^{N_{\tau}} \cos ^{2} \varphi_{\alpha}+\left(\varepsilon_{r}^{-1}+(-1)^{N_{\tau}} \varepsilon^{-1}\right) \cos \varphi_{\alpha}\right),
$$

где

$$
\begin{gathered}
R^{2}=\varepsilon_{r}^{-2}+\varepsilon^{-2}-1 \\
\varepsilon=\frac{1}{\operatorname{ch}\left(\frac{m}{T}\right)}, \quad \varepsilon_{r}=\frac{1}{\operatorname{ch}\left(\frac{m+2 m_{r}}{T}\right)} .
\end{gathered}
$$

Выражение (18) может быть переписано в виде

$$
e^{-\mathcal{S}_{\mathrm{F}}^{\mathrm{eff}}}=\prod_{\alpha=1}^{N}\left(\varepsilon_{r}^{-1}+(-1)^{N_{\tau}} \cos \varphi_{\alpha}\right)^{n_{f}}\left(\varepsilon^{-1}+\cos \varphi_{\alpha}\right)^{n_{f}} .
$$

Для калибровочной групшы $S U(2)$ имеем $\alpha=1,2$ и, следовательно, $\varphi_{\alpha}$ принимает значения $\left\{\varphi_{1}=\arccos (\chi / 2), \varphi_{2}=-\arccos (\chi / 2)\right\}$, где $\chi-$ характер фундаментального представления. Поэтому формула (21) может быть легко выражена в инвариантной форме

$$
e^{-\mathcal{S}_{\mathrm{F}}^{\mathrm{eff}}}=\left(\varepsilon_{r}^{-1}+(-1)^{N_{\tau}} \frac{\chi}{2}\right)^{2 n_{f}}\left(\varepsilon^{-1}+\frac{\chi}{2}\right)^{2 n_{f}} .
$$

В случае калибровочной группы $S U(3)$ эффективное действие $\mathcal{S}_{\mathrm{F}}^{\mathrm{eff}}$ выражается в терминах характеров фундаментального представления $\chi=\sum_{\alpha=1}^{3} \exp \left(i \varphi_{\alpha}\right)$ с помощью простого соотношения

$$
\begin{aligned}
\hat{J}(\varepsilon) \varepsilon^{3} & \equiv \prod_{\alpha=1}^{3}\left(1+\varepsilon \cos \varphi_{\alpha}\right)= \\
& =(1+\varepsilon)\left(1-\frac{\varepsilon}{2}\right)\left(1-\frac{\varepsilon}{2}+\varepsilon \operatorname{Re} \chi\right)+\frac{\varepsilon^{2}}{4} \chi \chi^{*}+\frac{\varepsilon^{3}}{4} \operatorname{Re} \chi^{2}
\end{aligned}
$$

\footnotetext{
3) В случае периодических граничных условий соответствующие формулы могут быть получены заменой $\varphi_{\alpha} \rightarrow \varphi_{\alpha}+\pi$.
} 
которое с точностью до несущественной константы приводит к выражению

$$
-\mathcal{S}_{\mathrm{F}}^{\mathrm{eff}}=n_{f} \ln \hat{J}\left((-1)^{N_{\tau}} \varepsilon_{r}\right)+n_{f} \ln \hat{J}(\varepsilon) .
$$

Как видно из формул (23) и (24), эффективное действие $\mathcal{S}_{\mathrm{F}}^{\mathrm{eff}}$ усиливает вклад конфигураций с $\operatorname{Im} \chi=0$ и $\operatorname{Re} \chi \sim \max \operatorname{Re} \chi=3$, что, в частности, приводит к явному нарушению $Z(3)$-инвариантности. Фермионньй вклад снимает $Z(N)$-вырождение, и только фаза, в которой поляковская петля действительна, остается стабильной. Другие же фазы метастабильны. Как видно из (24), нарушение $Z(N)$-симметрии становится слабее по мере увеличения $m / T$ и в этом случае

$$
-\mathcal{S}_{\mathrm{F}}^{\mathrm{eff}} \simeq(-1)^{B+1} e^{-\frac{m}{T}} 2 \operatorname{Re} \chi
$$

что согласуется с аргументами, приведенными в статье [16]. Термодинамические свойства метастабильных фаз при этом меняются. В частности, их время жизни увеличивается с ослаблением нарушения $Z(N)$-симметрии.

Отметим также, что в простой модели фермионный вклад может быть целиком включен в меру. Например, в случае $S U(2)$-калибровочной группы, когда $m_{r} / T \gg 1$, модифицированная мера с точностью до несущественной константы имеет следующий вид:

$$
d \tilde{\mu}_{n_{f}}=\left(1+\varepsilon \cos \frac{\varphi}{2}\right)^{2 n_{f}} \sin ^{2} \frac{\varphi}{2} d \varphi
$$

Для $S U(3)$-калибровочной группы мера может быть записана в виде

$$
d \tilde{\mu}_{n_{f}}=\left((1+\varepsilon)\left(1-\frac{\varepsilon}{2}\right)\left(1-\frac{\varepsilon}{2}+\varepsilon \operatorname{Re} \chi\right)+\frac{\varepsilon^{2}}{4} \chi \chi^{*}+\frac{\varepsilon^{3}}{4} \operatorname{Re} \chi^{2}\right)^{n_{f}} d \mu
$$

где

$$
d \mu=\delta\left(\sum_{\alpha} \varphi_{\alpha}\right) \prod_{\alpha} \sin ^{2} \frac{\varphi_{\alpha}-\varphi_{\alpha+1}}{2} d \varphi_{\alpha}
$$

\section{3. КИРАЛЬНЫЙ КОНДЕНСАТ}

Подынтегральное выражение в статистической сумме

$$
\mathcal{Z}=\int \exp \left(-\mathcal{S}_{\mathrm{F}}^{\mathrm{eff}}-\mathcal{S}_{\mathrm{G}}\right) d \mu
$$

где $\mathcal{S}_{\mathrm{G}}$ - глюонная часть действия КХД, имеет нули в комплексной $m$-плоскости при $\Xi_{\alpha}=0$. Принимая во внимание соотношение $(16)$, из условия $\Xi_{\alpha}=0$ находим для четных $N_{\tau}$

$$
\operatorname{ch}\left(N_{\tau} \operatorname{arsh}\left(\left(m+m_{r}\right) a_{\tau}\right)\right)=\operatorname{ch}\left(\frac{m_{r}}{T}+i \varphi_{\alpha}+i \pi\right) .
$$

Как упоминалось выше, функции $M_{N_{\tau}}$ являются полиномами Чебышева порядка $N_{\tau}$ от $\left(m+m_{r}\right) a_{\tau}$, поэтому уравнение $\Xi_{\alpha}=0$ имеет $N_{\tau}$ решений. Действительно, переписывая (30) в виде

$$
\operatorname{arsh}\left(\left(m+m_{r}\right) a_{\tau}\right)= \pm\left(m_{r} a_{\tau}+i \frac{\varphi_{\alpha}+(2 k+1) \pi}{N_{\tau}}\right)
$$


где $k$ - целое, находим

$$
m_{\alpha}^{(k)}= \pm \frac{1}{a_{\tau}} \operatorname{sh}\left(m_{r} a_{\tau}+i \frac{\varphi_{\alpha}+(2 k+1) \pi}{N_{\tau}}\right)-m_{r}
$$

Легко убедиться, что в случае нечетных $N_{\tau}$ мы снова приходим к условию (32).

При конечных $a_{\tau}$ и $N_{\tau}$ нули функции $\operatorname{det} \Delta^{\dagger} \Delta$ распределены в широкой области комплексной $m$-плоскости, а при $a_{\tau} \rightarrow 0$ и $N_{\tau} \rightarrow \infty$ они располагаются вдоль линий $\operatorname{Re} m=0$ и $\operatorname{Re} m=-2 m_{r}$ :

$$
m_{\alpha}^{(k)}= \pm i\left[\varphi_{\alpha}+(2 k+1) \pi\right] T, \pm i\left[\varphi_{\alpha}+(2 k+1) \pi\right] T-2 m_{r}
$$

Как известно, нули $m_{\alpha}^{(k)}$ могут быть сопоставлены собственным значениям $\lambda_{\alpha}^{(k)}=$ $-i m_{\alpha}^{(k)}$ решеточного оператора Дирака. Известное соотношение Бэнкса-Кашера [17] связьвает кварковый конденсат со спектральной плотностью евклидова оператора Дирака $\rho(\lambda)$

$$
\langle\bar{\psi} \psi\rangle=\int \frac{\rho(\lambda)}{m-i \lambda} d \lambda=\frac{T}{V_{\sigma}} \sum_{\{\lambda\}} \frac{1}{m-i \lambda},
$$

где $V_{\sigma}$ - пространственньй объем решетки.

Спектр безмассового оператора Дирака дискретный и обладает киральной и зарядовой симметриями. Поэтому собственные значения с необходимостью появляются в комбинациях $\left(\lambda, \lambda^{*},-\lambda,-\lambda^{*}\right)$. В случае оператора Вильсона-Дирака $D_{x^{\prime} x}^{0}$ киральная симметрия $\left(\lambda \longleftrightarrow \lambda^{*}\right)$ нарушена, однако зарядовая симметрия $\left(\varphi_{\alpha} \longleftrightarrow-\varphi_{\alpha}\right)$ гарантирует, что собственные значения $\lambda_{\alpha}^{(k)}=-i m_{\alpha}^{(k)}$ появляются парами $\left(\lambda_{\alpha}^{(k)},-\lambda_{\alpha}^{(-k-1) *}\right)$. С учетом (33) выражение (34) может быть переписано в виде

$$
\langle\bar{\psi} \psi\rangle=\frac{n_{f}}{2} \sum_{\alpha=1}^{N} \sum_{k=-\infty}^{\infty}\left\langle\frac{1}{\frac{m}{T}-i\left(\varphi_{\alpha}+(2 k+1) \pi\right)}\right\rangle+\text { c.c. }
$$

где множитель $1 / 2$ включен для того, чтобы исключить двойной учет нулей (совпадающих вследствие зарядовой симметрии). Скобки в формуле (35) означают вычисление обычного среднего, т.е. суммирование по $\varphi_{\alpha}$ с весом, определяемым действием КХД.

Можно записать [18, гл. $5, \S 1$, разд. 25 , формула (3)], что

$$
\sum_{k=-\infty}^{\infty} \frac{1}{\left(\frac{m}{T}\right)^{2}+\left(\varphi_{\alpha}+\pi+2 \pi k\right)^{2}}=\frac{T}{m} \frac{\operatorname{sh} \frac{m}{T}}{\operatorname{ch} \frac{m}{T}+\cos \varphi_{\alpha}}
$$

откуда окончательно получаем

$$
\langle\bar{\psi} \psi\rangle=n_{f} \operatorname{sh} \frac{m}{T} \sum_{\alpha=1}^{N}\left\langle\frac{1}{\operatorname{ch} \frac{m}{T}+\cos \varphi_{\alpha}}\right\rangle+\left(m \rightarrow m+2 m_{r}\right)
$$


Полученная формула, как и следовало ожидать, согласуется со стандартным определением для кирального конденсата

$$
\langle\bar{\psi} \psi\rangle=\frac{T}{V} \frac{\partial \ln Z}{\partial m}
$$

если фермионный вклад представлен эффективным действием (21).

Весьма любопытно, что положение нулей не фиксировано и они распределены в $m$ плоскости с весом, определяемым эффективным глюонным $\mathcal{S}_{\mathrm{G}}^{\mathrm{eff}}\left(\varphi_{\alpha}\right)$ и фермионным $\mathcal{S}_{\mathrm{F}}^{\mathrm{eff}}\left(\varphi_{\alpha}\right)$ действиями, которые получаются в результате интегрирования по всем переменным, за исключением поляковских петель $\Omega_{\alpha \nu}=\delta_{\alpha \nu} e^{i \varphi_{\alpha}}$ в одной точке. Иными словами,

$$
\rho(\lambda) d \lambda=\exp \left\{-\mathcal{S}_{\mathrm{G}}^{\mathrm{eff}}\left(\varphi_{\alpha}\right)-\mathcal{S}_{\mathrm{F}}^{\mathrm{eff}}\left(\varphi_{\alpha}\right)\right\} d \mu\left(\varphi_{\alpha}\right)
$$

Чтобы оценить $-\mathcal{S}_{\mathrm{G}}^{\text {eff }}$, необходимо в духе метода Кюри-Вейсса заменить все линковые переменные, за исключением входяших в поляковскую линию, средним полем $H$. Например, в случае $S U(2)$-калибровочной группы $\left(\varphi_{1}=-\varphi_{2}=\varphi / 2\right)$ это приводит к соотношению

$$
-\mathcal{S}_{\mathrm{G}}^{\mathrm{eff}}(\varphi) \simeq H 2 \cos \frac{\varphi}{2}
$$

поэтому

$$
\rho(\lambda) d \lambda \simeq e^{H 2 \cos \frac{\varphi}{2}}\left(\frac{1}{\varepsilon}+\cos \frac{\varphi}{2}\right)^{2 n_{f}}\left(\frac{1}{\varepsilon_{r}}+\cos \frac{\varphi}{2}\right)^{2 n_{f}} \sin ^{2} \frac{\varphi}{2} \frac{d \varphi}{2 \pi}
$$

Следовательно, можно сделать вывод, что, по крайней мере, в данном приближении нулевые моды $(\lambda=0 \longleftrightarrow \varphi / 2= \pm \pi)$ сильно подавлены.

Попытаемся теперь исследовать поведение кирального конденсата в простой модели в некоторых предельных случаях. С учетом (37) можно записать для случаев $m / T \gg 1$ и $m_{r} / T \gg 1$, что

$$
\langle\bar{\psi} \psi\rangle=2-\left(\frac{(-1)^{N_{\tau}}}{\operatorname{ch} \frac{m}{T}}+\frac{1}{\operatorname{ch} \frac{m+2 m_{r}}{T}}\right)\langle\operatorname{Re} \chi\rangle .
$$

В области $m / T \ll 1$ и $m_{r} / T \ll 1$ выражение $(37)$ может быть записано в виде

$$
\langle\bar{\psi} \psi\rangle \simeq \frac{m}{T}\left\langle\frac{1}{1+\cos \frac{\varphi}{2}}\right\rangle+\frac{m+2 m_{r}}{T}\left\langle\frac{1}{1+(-1)^{N_{\tau} \cos \frac{\varphi}{2}}}\right\rangle .
$$

Для четных $N_{\tau}$ грубые оценки приводят к формуле

$$
\langle\bar{\psi} \psi\rangle \simeq \frac{m+m_{r}}{T}\left\langle\frac{2}{1+\cos \frac{\varphi}{2}}\right\rangle \sim \frac{m+m_{r}}{T} .
$$

Одно из важных свойств кирального конденсата - поведение среднего $\langle\bar{\psi} \psi\rangle$ при асимптотически малых массах кварков $m$. Хорошая подгонка экспериментальных данных (в 
симметричной фазе КЭД те [19] выражением

$$
\langle\bar{\psi} \psi\rangle \ln ^{\varepsilon} \frac{1}{\langle\bar{\psi} \psi\rangle} \sim m, \quad \varepsilon \sim \frac{1}{4} .
$$

Сходные результаты были получены в статье [20] для $S U(3)$-калибровочной группы в случае $n_{f}=2$ на решетке $N_{\tau}=6$. Легко проверить, что данные [20] согласуются с формулой

$$
\langle\bar{\psi} \psi\rangle \simeq 6 a m=\frac{m}{T} .
$$

Наша оценка для $\langle\bar{\psi} \psi\rangle$, представленная формулой (44), грубо согласуется с (45) и (46), только когда $m_{r} \sim m$. Наконец, хотелось бы отметить, что в рассмотренном приближении киральная симметрия может быть восстановлена, только если $m / T \rightarrow 0$ и $m_{r} / T \rightarrow 0$. Однако, чтобы устранить вклад зеркальных фермионов, необходимо устремить $m_{r}$ к бесконечности. При этом, например в (13) или (32), могут возникнуть неоднозначности при переходе к континуальному пределу. Избежать этого можно, переходя к пределу $m_{r} \rightarrow \infty$ и $a_{\tau} \rightarrow 0$ согласованно. Если параметр $m_{r}$ стремится к бесконечности настолько медленно, что $m_{r} a_{\tau} \rightarrow 0$, можно записать ${ }^{4)}$

$$
-\mathcal{S}_{\mathrm{F}}^{\mathrm{eff}}=-\mathcal{S}_{\mathrm{mir}}+n_{f} \sum_{\alpha=1}^{N} \ln \left(\cos \frac{m}{T}+\cos \varphi_{\alpha}\right),
$$

где

$$
-\mathcal{S}_{\text {mir }}=n_{f} \ln \operatorname{ch}\left(m+\frac{2 m r}{T}\right) \simeq \frac{N n_{f} m}{T}+\text { const } .
$$

$\mathrm{K}$ сожалению, при быстром росте $m_{r}$, когда $m_{r} a_{\tau}$ остается конечным, разложение по степеням $a_{\tau}$ в $(12)$ становится недопустимым, и этот случай следует рассмотреть особо. Чтобы убедиться в том, что результат не зависит от способа стремления $m_{r}$ к бесконечности, можно рассмотреть предельньй случай, когда $m_{r} a_{\tau}$ в континуальном пределе неограниченно растет, что, как это видно из (11), соответствует выбору $r=1$. Вычисление детерминанта в этом случае значительно упрошается. Действительно,

$$
\Delta_{x x^{\prime}}=\delta_{\vec{x} \vec{x}^{\prime}}\left(U_{0}(\vec{x}, t) \delta_{t, t^{\prime}-1}-\left(1+m a_{\tau}\right) \delta_{t, t^{\prime}}\right)
$$

и с помошью соотношения (63) из приложения находим

$$
\operatorname{det} \Delta=\prod_{\alpha=1}^{N}\left(e^{\frac{m}{T}}+e^{i \varphi_{\alpha}}\right),
$$

что, как легко проверить, снова ведет к формуле (47) с тем лишь отличием, что соотношение

$$
-\mathcal{S}_{\mathrm{mir}}=N n_{f} \frac{m}{T}
$$

\footnotetext{
${ }^{4)}$ Отметим, что в пределе $m_{r} \rightarrow \infty$ различие результатов для решеток с четным и нечетным $N_{\tau}$ исчезает.
} 
выполняется точно

Легко убедиться, что именно этот член дает нежелательный вклад в среднее $\langle\bar{\psi} \psi\rangle$, не исчезающий даже при нулевых массах обычных фермионов. К сожалению, мы можем предложить устранение этого вклада лишш путем добавления в исходное действие (1) "контрчлена" $\mathcal{S}_{\mathrm{F}}=\mathcal{S}_{\mathrm{F}}-\mathcal{S}_{\mathrm{mir}}$, сохраняя при этом для $\langle\bar{\psi} \psi\rangle$ стандартное определение (38). Такой прием, очевидно, устраняет нежелательный вклад в $\langle\bar{\psi} \psi\rangle$, и для кирального конденсата мы можем в этом случае записать для $m / T \ll 1$ соотношение

$$
\langle\bar{\psi} \psi\rangle=n_{f} \sum_{\alpha=1}^{N}\left\langle\left(1+\frac{\cos \varphi_{\alpha}}{\operatorname{ch} \frac{m}{T}}\right)^{-1}\right\rangle \text { th } \frac{m}{T} \simeq \frac{m}{T} n_{f} \sum_{\alpha}\left\langle\left(1+\cos \varphi_{\alpha}\right)^{-1}\right\rangle .
$$

При $m / T \gg 1$ для случая $S U(2)$ можно воспользоваться выражением

$$
\frac{1}{\operatorname{ch} x+\cos \frac{\varphi}{2}}=2 \sum_{j=0}^{\infty}(-1)^{2 j} \chi_{j}(\varphi) e^{-(2 j+1)}|x|,
$$

где $\chi_{j}(\varphi)$ - характеры неприводимых представлений $S U(2)$, и записать

$$
\langle\bar{\psi} \psi\rangle=4 n_{f} \operatorname{sh} \frac{m}{T} \sum_{j=0}^{\infty} e^{-(2 j+1) \frac{m}{T}}(-1)^{2 j}\left\langle\chi_{j}\right\rangle .
$$

\section{4. ОБСУЖДЕНИЕ}

В доступной для современных компьютеров области параметров вклад кваркового моря не вносит существенных изменений в монте-карловские данные. Например, сравнение спектроскопических результатов, полученных с динамическими и "quenched"фермионами, не обнаруживает серьезных различий. По-видимому, при выбранных для симуляций значениях параметров поправки, вносимые в спектры морем кварков, не превышают 5-10\% [20]. Это позволяет считать, что “физика” легких динамических кварков сходна с “физикой” quenched-кварков той же массы [21]. В настоящее время монте-карловский эксперимент скорее позволяет обнаружить эффекты, вносимые кварковым морем [21-23], нежели указать, в какой области параметров эти эффекты окажутся существенными.

Ситуация может измениться, когда техника монте-карловских вычислений позволит изучать область малых кварковых масс при малом решеточном шаге. Даже при ограниченных возможностях современных компьютеров удается показать, что в некоторых областях параметров вклад динамических кварков оказывается значительным. Так, в работе [24] влияние динамических фермионов изучалось в фазе конфайнмента путем сравнения КЭД с вильсоновскими и quenched-фермионами. Было установлено, что в пределе сильной связи $(\beta=0)$ теория quenched-фермионов может служить хорошим приближением полной теории, но полностью ей не соответствует при $\beta=0.8$.

Мы надеемся, что предложенная в этой работе модель не только поможет оценить вклад кваркового моря на решетках размеров, недоступных для монте-карловского эксперимента, но и позволит определить область параметров, где присутствие динамических фермионов приводит к наиболее заметным эффектам. 
Наконец приведем некоторые доводы в пользу того, что периодичность спектра дираковского решеточного оператора может иметь место не только в пределе $\xi \gg 1$, но и в более общем случае. Как аналитические исследования [25-27], так и численный эксперимент [28-33] указывают на то, что собственные значения дираковского оператора сосредоточены в компактной области. Во многих случаях, игнорируя незначительные формальные различия, компактность можно рассматривать как периодичность. Помимо этого, в конечнотемпературной теории периодичность спектра может индуцироваться периодичностью (антипериодичностью) переменных во временном направлении. Действительно, фермионная часть действия периодична по переменной $\varphi=\arg \Omega=$ $A_{0} / T$, что ведет к периодичности собственных значений оператора Дирака. Рассмотрим в качестве примера спектр свободного оператора Дирака [33]

$$
\lambda_{k}^{2}=\vec{p}^{2}+\left(\varphi_{\alpha}+\pi+2 \pi k\right)^{2} T^{2}
$$

где $\varphi_{\alpha}=0, \pm 2 \pi / 3$. Легко показать, что

$$
\langle\bar{\psi} \psi\rangle=\frac{m}{T} \sum_{k}\left\langle\frac{1}{\varepsilon^{2}+\left(\varphi_{\alpha}+\pi+2 \pi k\right)^{2}}\right\rangle=\frac{m}{T}\left\langle\frac{\operatorname{sh} \varepsilon}{\operatorname{ch} \varepsilon+\cos \varphi_{\alpha}}\right\rangle,
$$

где $\varepsilon=\sqrt{m^{2}+\vec{p}^{2}} / T$.

Убедительные и достаточно строгие доводы в пользу периодичности собственных значений оператора Дирака даны в работах [34-36], где показано, что если дираковский оператор удовлетворяет соотношению Гинспарга-Вильсона [36], то его собственные значения располагаются на окружности $\left(1-e^{i \theta}\right) / a, 0 \leqslant \theta<2 \pi$. Хотя приведенные аргументы едва ли могут быть приняты как доказательство, они дают серьезные основания надеяться, что собственные значения оператора Дирака периодичны в общем случае.

\section{5. ВЫВОДЫ}

Предложенный подход к проблеме оценки влияния динамических фермионов основан на исключении из рассмотрения всех членов действия порядка $1 / \xi$. Модель допускает аналитическое решение для произвольно малых значений решеточного шага и бесконечно больших решеток, т.е. в тех случаях, когда методы Монте-Карло становятся трудновыполнимыми и дорогостоящими. При нулевом химическом потенциале $\mu$ фермионный детерминант действителен и может быть включен в меру. Несмотря на то что его вклад достаточно тривиален, он значительно меняет фазовую структуру теории. Причем эти изменения весьма сходны (в особенности в случае тяжелых кварков) с теми, которые вносит включение внешнего поля в спиновых системах. Считается, что киральная симметрия, нарушенная вильсоновским членом, восстанавливается в континуальном пределе. В рассмотренной модели, однако, подобное восстановление не наблюдается ни при каких конечных значениях $m / T$ и $m_{r} / T$, причем киральная симметрия не может быть восстановлена “подстройкой” голых параметров (что противоречит предположению, сделанному в работе [24]). 
Хотелось бы также подчеркнуть сушественное различие результатов, полученных на решетках с четным и нечетным $N_{\tau}$ (при конечных $m_{r}$ ) (см. также [37]), исчезаюшее лишь при $m_{r} \rightarrow \infty$, и неожиданно малое различие между периодическими и антипериодическими граничными условиями.

Впервые прямое вычисление термодинамических величин КХД при малых кварковых массах было выполнено достаточно давно [38]. Тогда была установлена существенная разница между периодическими и антипериодическими граничными условиями. Однако эта разница, как подчеркивалось в [38], исчезала с увеличением решеточного объема. Хотя, как это видно из формул (22) и (24), различие между периодическими и антипериодическими граничными условиями формально не исчезает при любых $N_{\tau}$, однако, как видно из $(21)$, фактор $(-1)^{B}$ в случае четных $N$ может быть поглощен $Z(N)$-преобразованием. Наши оценки для случая $N=3$ показывают, что заметное различие между периодическими и антипериодическими граничными условиями возникает лишш в области $m / T \ll 1$.

Наконец заметим, что при $m_{r} \rightarrow \infty$ исчезает нежелательная симметрия $m \rightarrow$ $m+2 m_{r}$, которой спектр оператора Дирака не обладает. Однако симметрия $\lambda \rightarrow-\lambda$ (формально соответствуюшая $m \rightarrow-m$ ) восстанавливается лишь после добавления “контрчлена" в (1), что попросту соответствует отбрасыванию остаточного вклада зеркальных фермионов $\mathcal{S}_{\text {mir }}$ в $(47)$.

ПРИЛОЖЕНИЕ

Разлагая

$$
F_{n+1}=\left(\begin{array}{cccccc}
q_{n+1} & b_{n} & 0 & \ldots & 0 & c_{n+1} \\
c_{n} & q_{n} & b_{n-1} & \ldots & 0 & 0 \\
0 & c_{n-1} & q_{n-1} & \ldots & 0 & 0 \\
\ldots & \ldots & \ldots & \ldots & \ldots & \ldots \\
0 & 0 & 0 & \ldots & q_{2} & b_{1} \\
b_{n+1} & 0 & 0 & \ldots & c_{1} & q_{1}
\end{array}\right)
$$

по элементам первой строки

$$
F_{n+1}=q_{n+1} f_{n}+b_{n} \frac{\partial}{\partial b_{n}} F_{n+1}+c_{n+1} \frac{\partial}{\partial c_{n+1}} F_{n+1},
$$

получаем

$$
b_{n} \frac{\partial}{\partial b_{n}} F_{n+1}=-b_{n} c_{n} f_{n-1}-\prod_{j}^{n+1}\left(-b_{j}\right)
$$

$$
c_{n+1} \frac{\partial}{\partial c_{n+1}} F_{n+1}=-c_{n+1} b_{n+1} f_{n}^{(2)}-\prod_{j=1}^{n+1}\left(-c_{j}\right),
$$

где

$$
\begin{gathered}
f_{n}=q_{n} f_{n-1}-b_{n-1} c_{n-1} f_{n-2}=F_{n} \quad\left(b_{n}=c_{n}=0\right), \\
f_{n}^{(2)}=\frac{\partial}{\partial q_{n+1}} \frac{\partial}{\partial q_{1}} F_{n+1} .
\end{gathered}
$$


Рассмотрим два простых случая. Пусть сначала $c_{j}=0$. Из соотношений $(58)$ и (60) следует, что

$$
b_{n} \frac{\partial F_{n+1}}{\partial b_{n}}=-\prod_{j=1}^{n+1}\left(-b_{j}\right), \quad f_{n}=q_{n} f_{n-1}=\prod_{j=1}^{n} q_{j},
$$

т.e.

$$
F_{n}=\prod_{j=1}^{n} q_{j}-\prod_{j=1}^{n}\left(-b_{j}\right) .
$$

В другом простом случае, когда $q_{j}$ и $b_{j} c_{j}$ не зависят от $j$,

$$
q_{j}=q, \quad b_{j}=\frac{\lambda}{\omega_{j}}, \quad c_{j}=-\lambda \omega_{j}
$$

можно записать

$$
f_{j}^{(2)}=f_{j-1}
$$

$$
f_{j+1}-q f_{j}-\lambda^{2} f_{j-1}=0 .
$$

Уравнение (66) легко решить, введя производящую функцию

$$
f(z)=\sum_{j=n_{0}}^{\infty} f_{j} z^{j-1}
$$

что приводит к уравнению

$$
\sum_{j=n_{0}}^{\infty}\left(\frac{1}{z} f_{j+1} z^{j+1}-q f_{j} z^{j}-\lambda^{2} z f_{j-1} z^{j-1}\right)=\left(\frac{1}{z}-q-\lambda^{2} z\right) f(z)=0
$$

где $n_{0}$ - произвольное фиксированное число. В частности, для $n_{0}=2$ и $n_{0}=3$ из (66) легко получить

$$
f_{2}=q^{2}+\lambda^{2}, \quad f_{3}=q^{3}+2 \lambda^{2} q,
$$

следовательно, общее решение уравнения (66) может быть записано в виде

$$
f_{n}=\frac{f_{n_{0}}}{2 \pi i} \int \frac{z^{-n-1} d z}{\frac{1}{z}-q-\lambda^{2} z}=\left\{\begin{array}{lll}
2 \lambda^{n} \operatorname{ch}\left(n \operatorname{arsh} \frac{q}{2 \lambda}\right) & \text { при } \quad n=2 k, \\
2 \lambda^{n} \operatorname{sh}\left(n \operatorname{arsh} \frac{q}{2 \lambda}\right) & \text { при } \quad n=2 k+1 .
\end{array}\right.
$$

Окончательно находим для $F_{n}$ выражение

где

$$
\frac{F_{n}}{2 \lambda^{n}}= \begin{cases}\operatorname{ch}\left(n \operatorname{arsh} \frac{q}{2 \lambda}\right)-\frac{\frac{1}{\Omega}+\Omega}{2} & \text { при } n=2 k, \\ \operatorname{sh}\left(n \operatorname{arsh} \frac{q}{2 \lambda}\right)-\frac{\frac{1}{\Omega}-\Omega}{2} & \text { при } n=2 k+1,\end{cases}
$$

$$
\Omega=\prod_{j=1}^{n} \omega_{j} .
$$

\section{Список литературы}

[1] V. K. Petrov. Scaling in a toy model of gluodynamics at finite temperatures; hep-lat/9803019.

[2] Л. А. Аверченкова, Г. М. Зиновьев, В. К. Петров, К. В. Петров. ЯФ. 1997. Т. 60. № 11. C. 2057.

[3] M. Billo, M. Casselle, A. D’Adda, S. Panzeri. Nucl. Phys. B. 1996. V. 472. P. 163. 
[4] F. Green, F. Karsch. Nucl. Phys. B. 1984. V. 238. P. 297.

[5] M. Ogilvie. Phys. Rev. Lett. 1984. V. 52. P. 1369.

[6] A. Ukawa. Nucl. Phys. Proc. Suppl. 1997. V. 53. P. 106.

[7] Y. Iwasaki. Nucl. Phys. Proc. Suppl. 1995. V. 42. P. 96.

[8] H. B. Nielsen, H. Ninomiya. Nucl. Phys. B. 1981. V. 185. P. 20; 1982. V. 195. P. 541.

[9] K. M. Bitar, U. M. Heller, R. Narayanan. Phys. Lett. B. 1998. V. 418. P. 167.

[10] P. Hasenfratz, F. Karsch. Phys. Lett. B. 1983. V. 125. P. 308

[11] I. Bender, H. J. Rothe, W. Wetzel, I. O. Stamatescu. Z. Phys. C. 1993. V. 58. P. 333.

[12] A. А. Славнов, Л. Д. Фаддеев. Введение в квантовую теорию калибровочных полей. М.: Наука, 1978

[13] K. Osterwalder, R. Schrader. Commun. Math. Phys. 1973. V. 31. P. 83; 1975. V. 42 . P. 281.

[14] K. Osterwalder, E. Seiler. Ann. Phys. 1978. V. 110. P. 440.

[15] Г. Бәйтмен, А. Эрдейи. Высшие трансцендентные функции. М.: Наука, 1974.

[16] Peter N. Meisinger, Michael C. Ogilvie. Phys. Lett. B. 1996. V. 379. P. 163.

[17] T. Banks, A. Casher. Nucl. Phys. B. 1980. V. 169. P. 103.

[18] А. П. Прудников, Ю. А. Брычков, О. И. Маричев. Интегралы и ряды. М.: Наука, 1981.

[19] V. Azcoiti, G. Di Carlo, A. Galante, A. F. Grillo, V. Laliena, C. Piedrafita. Nucl. Phys. Proc. Suppl. 1996. V. 47. P. 659.

[20] C. Bernard, T. Blum, C. DeTar, S. Gottlieb, U. M. Helle, J. E. Hetrick, K. Rummukainen, R. Sugar, D. Toussaint, M. Wingate. Phys. Rev. Lett. 1997. V. 78. P. 598.

[21] A. D. Kennedy, R. G. Edward. On the dynamics of light Wilson quarks. hep-lat/9201007.

[22] S. Collins, R. G. Edwards, U. M. Heller, J. Sloan. Nucl. Phys. Proc. Suppl. 1996. V. 47. P. 378.

[23] A. Spitz, N. Eicker, J. Fingberg, S. Güsken, H. Hoeber, Th. Lippert, K. Schilling, J. Viehoff. Nucl. Phys. Proc. Suppl. 1998. V. 63. P. 317.

[24] A. Hoferichter, V. K. Mitryushkin, M. Muller-Preussker, H. Stuben. Phys. Rev. D. 1998. V. 58. P. 114505.

[25] M. Luscher. Nucl. Phys. B. 1994. V. 418. P. 637; hep-lat/9311007.

[26] B. Bunk, K. Jansen, B. Jegerlehner, M. Luscher, H. Simma, R. Sommer. Nucl. Phys. Proc. Suppl. 1995. V. 42. P. 49; hep-lat/9411016.

[27] K. Jansen, C. Liu, H. Simma, D. Smith. Nucl. Phys. Proc. Suppl. 1997. V. 53. P. 262; hep-lat/9608048.

[28] T. Kalkreuter. Comput. Phys. Commun. 1996. V. 95. P. 1; hep-lat/9509071.

[29] T. Kalkreuter. Nucl. Phys. Proc. Suppl. 1995. V. 42. P. 882; hep-lat/9410006.

[30] T. Kalkreuter. Phys. Rev. D. 1995. V. 51. P. 1305; hep-lat/9408013.

[31] J. C. Osborn, J. J. M. Verbaarschot. Thouless Energy and Correlations of QCD Dirac Eigenvalues. hep-ph/9803419.

[32] A. V. Smilga. Chiral symmetry and spectrum of Euclidean Dirac operator in QCD. hep-th/9503049.

[33] M. A. Stephanov. Phys. Lett. B. 1996. V. 375. P. 249; Chiral Symmetry at Finite T, the Phase of the Polyakov Loop and the Spectrum of the Dirac Operator. hep-lat/9601001.

[34] H. Neuberger. More about exactly massless quarks on the lattice. hep-lat/9801031.

[35] R. Narayanan. Ginsparg-Wilson relation and the overlap formula. hep-lat/9802018.

[36] K. Splittorff, A. D. Jackson. The Ginsparg-Wilson relation and local chiral random matrix theory. hep-th/980518.

[37] O. A. Borisenko, V. K. Petrov, G. M. Zinovjev. Nucl. Phys. B. 1995. V. 437. P. 391.

[38] I. M. Barbour, J. P. Gilchrist, H. Schneider, G. Schierholz, M. Teper. Phys. Lett. B. 1983. V. 127. P. 433.

Поступила в редакцию 3.III.1999 г., после доработки 1.IX.1999 г. 